\title{
Effect of Nonsurgical Periodontal Therapy on Some Oxidative Stress Markers in Patients with Chronic Periodontitis: A Biochemical Study
}

\author{
Abdul S Aziz, Madhav G Kalekar, Tabita Benjamin, Adinath N Suryakar, Milsee M Prakashan
}

Mohammed Nadeem A Bijle

\begin{abstract}
Introduction: Chronic periodontitis is the inflammatory disease of the periodontium; the tissue that surround and support the teeth. The aim of the study was to estimate and compare some biochemical oxidative stress markers in patients with chronic periodontitis and healthy controls and to assess the effect of nonsurgical periodontal therapy on these parameters.
\end{abstract}

Materials and methods: Eighty-two chronic periodontitis patients and 120 healthy controls were recruited for the study. The periodontal status was evaluated using gingival index (GI), plaque index (PI), papillary bleeding index (PBI) and clinical attachment loss (CAL). The biochemical parameters estimated were total antioxidant capacity (TAOC), antioxidant enzymes RBC-superoxide dismutase (RBC-SOD) and glutathione peroxidase (GPx), vitamin $\mathrm{C}$, malondialdehyde (MDA) and Creactive protein (CRP) were also evaluated. Nonsurgical periodontal therapy (scaling and root planing; SRP) was performed on chronic periodontitis patients and a follow-up was done after 3 months.

Results: The study suggests that oxidative stress is induced in chronic periodontitis, as the levels of CRP, MDA and RBC-SOD were significantly higher $(p<0.001)$ and those of TAOC, GPX and vitamin $C$ were significantly lower $(p<0.001)$ compared to healthy controls at baseline. SRP therapy significantly improved the clinical and biochemical oxidative stress markers in periodontitis patients.

Conclusion: Chronic periodontitis patients show higher inflammatory manifestations and oxidative stress. SRP therapy helps in lowering inflammatory burden and improving systemic oxidant: Antioxidant imbalance. The SRP therapy may be a useful support in the control and prevention of various inflammatory diseases associated with periodontitis.

Keywords: Chronic periodontitis, Oxidative stress, Nonsurgical periodontal therapy, Biochemical oxidative stress markers.

How to cite this article: Aziz AS, Kalekar MG, Benjamin T, Suryakar AN, Prakashan MM, Bijle MNA. Effect of Nonsurgical Periodontal Therapy on Some Oxidative Stress Markers in Patients with Chronic Periodontitis: A Biochemical Study. World J Dent 2013;4(1):17-23.

Source of support: Nil

Conflict of interest: None declared

\section{INTRODUCTION}

Periodontitis, which affects the supporting tissues of teeth, is an inflammatory disorder of the periodontium. ${ }^{1}$ It is initiated by the subgingival biofilm but the progression of destructive disease appears to be dependent upon an abnormal host response to these organisms. ${ }^{2}$ Periodontal disease is regarded as a risk factor for the development of various systemic conditions, such as cardiovascular disease, diabetes, osteoporosis and adverse pregnancy outcomes. ${ }^{3}$ Oxidative stress is an imbalance between pro-oxidants and antioxidants in favor of the former. It has been related increasingly to the onset and/or progression of growing number of human diseases. ${ }^{4}$ Oxidative stress has been linked with both onset of periodontal tissue destruction and systemic inflammation. ${ }^{5}$ Reactive oxygen species (ROS) such as superoxide anion, hydroxyl radical, nitrous oxide and hydrogen peroxides are produced via the bacteria-host mediated pathway, stimulating polymorphonuclear leukocytes (PMNL) to produce superoxide radicals via 'respiratory burst'. The increased ROS concentration leads to oxidative damage to periodontal tissues with an impaired circulating oxidant: Antioxidant balance. ${ }^{6,7}$

All mammalian cells contain antioxidants that prevent or limit oxidative tissue injury caused by ROS. ${ }^{8}$ Antioxidants may be enzymes, such as superoxide dismutase (SOD), catalase (CAT), etc. high molecular weight proteins, such as albumin, ceruloplasmin, etc. low molecular weight water soluble antioxidants such as vitamin C, uric acid, etc. and low molecular weight water insoluble antioxidants such as tocopherol, carotenoids, etc. Antioxidants overall, act at different levels of action such as prevention, interception and repair damage caused by ROS. In a normal healthy human body the generation of free radicals or pro-oxidants is effectively kept in check at various levels by antioxidant defense mechanism. However, when it gets exposed to adverse physicochemical, environmental or pathological agents, this delicately maintained balance (oxidant: Antioxidant) shifts in favor of pro-oxidants, resulting in oxidative stress. ${ }^{9,10}$

Studies in the literature have reported the beneficial effects of scaling and root planing (SRP) therapy on some key local markers, ${ }^{11}$ some systemic oxidative stress markers ${ }^{1}$ and systemic inflammatory markers ${ }^{12}$ independently. However, few studies have addressed the effect of periodontal therapy on local clinical, systemic oxidative stress markers in conjunction with systemic inflammatory 
markers. Therefore, the present study assesses the effect of SRP therapy on clinical and some biochemical oxidative stress marker and inflammatory marker in patients with chronic periodontitis.

\section{MATERIALS AND METHODS}

The study was undertaken as per the approval of the Institutional Ethics Committee of Grant Medical College and Sir JJ Group of Hospitals, Mumbai, following norms of the World Medical Association of Helsinki; ethical principles for medical research involving human subjects (amended by 55th WMA General Assembly, 2004). A written consent was obtained from all the subjects enrolled in the study. The subjects had the right to refuse to participate in the study or to withdraw consent to participate at any time without reprisal.

\section{Study Groups}

A total 82 patients with chronic periodontitis (group II), who visited the Department of Dentistry Grant Medical College, Mumbai, and 120 apparently healthy volunteers (group I) were recruited for the study. The distribution of sex and age between the groups are shown in Table 1. The patients in the study group were clinically evaluated for chronic periodontitis according to the criteria accepted by the American Academy of Periodontology in $1999^{13}$ and the periodontal status was evaluated as described by Armitage (2004). ${ }^{14}$ The patients were otherwise healthy, with no history of major illness and consumption of antioxidants, antibiotics, anti-inflammatory or any other drugs and had not received any periodontal therapy for at least 6 months prior to the inception of the study. Subjects who had past illness and undergoing any treatment, diabetics, smokers and tobacco consumers, alcoholics, pregnant and lactating women were excluded from the study. The individuals in the group I were from the same geographical region and similar socioeconomic status with apparently good oral and systemic health.

\section{Periodontal Therapy}

The group II patients received periodontal therapy, which included SRP and oral hygiene instructions. The instructions included demonstration of correct brushing technique and information to brush twice daily after meals. The SRP was performed by qualified periodontologist using ultrasonic instrument (Electro Medical System, Switzerland) and manual Gracey curettes (Hu-Friedy, Avco). The patients were monitored periodically, were followed up after 3 months and their clinical parameters were evaluated and blood samples were collected. Subjects in group I received no periodontal treatment during the course of the study and were evaluated only once.

\section{Clinical Measurements}

The periodontal status of all individuals was evaluated by measurement of gingival index (GI) as developed by Loe and Silness (1963), ${ }^{15}$ plaque index (PI) as described by Silness and Loe (1964), ${ }^{16}$ papillary bleeding index (PBI) developed by Muhlemann (1977) ${ }^{17}$ and clinical attachment loss (CAL). CAL measurements are recorded at six sites around each tooth (i.e. mesiobuccal, buccal, distobuccal, mesiolingual, lingual and distolingual) from the cementoenamel junction to the base of the pocket. ${ }^{14}$ All clinical measurements were evaluated by a single trained investigator using University of Carolina (UNC-15) probe. The investigator was blinded to the individuals of both the groups.

\section{Sample Collection}

A total of $4 \mathrm{ml}$ venous blood was collected in disposable syringe from all the subjects following standard precautionary measures. Of this, $1 \mathrm{ml}$ heparinized blood was used for analysis of total antioxidant capacity (TAOC), RBC-SOD and glutathione peroxidase (GPx). The remaining $3 \mathrm{ml}$ of blood was allowed to stand at room temperature for 30 minutes and then centrifuged at 3,000 rpm for 20 minutes to obtain serum, which was stored at $-4^{\circ} \mathrm{C}$ until further processing. The serum was analyzed of vitamin C, malondialdehyde (MDA) and C-reactive protein (CRP). The blood sample was collected twice once at baseline and then after 3 months for patients in group II and only once for subjects in group I.

Table 1: Distribution of age and sex between the study groups

\begin{tabular}{|c|c|c|c|c|c|}
\hline \multirow[t]{2}{*}{ Parameters } & & \multicolumn{2}{|c|}{ Group I $(n=120)$} & \multicolumn{2}{|c|}{ Group II $(n=82)$} \\
\hline & & Number of subjects (n) & $\%$ of subjects & Number of subjects ( $n$ ) & $\%$ of subjects \\
\hline Sex & Male & 64 & 53.3 & 49 & 59.8 \\
\hline & Female & 56 & 46.7 & 33 & 40.2 \\
\hline $\begin{array}{l}\text { Age (years) } \\
\text { Mean }\end{array}$ & \multicolumn{3}{|c|}{$39.6 \pm 8.4$} & \multicolumn{2}{|c|}{$41.6 \pm 6.4$} \\
\hline
\end{tabular}




\section{Biochemical Studies}

\section{TAOC Assay}

The plasma TAOC was measured by the ferric reducing ability of plasma (FRAP) assay according to the method of Benzie and Strain. ${ }^{18}$ The reaction measures antioxidant reduction of $\mathrm{Fe}^{3+} \mathrm{TPTZ}$ (tripyridyl triazine) to blue colored $\mathrm{Fe}^{2+}$ TPTZ. FRAP values were obtained by comparing the absorbance change at $593 \mathrm{~nm}$ in test reaction mixture with those containing ferrous ions in known concentration.

\section{RBC-SOD Activity Assay}

The RBC-SOD was measured using the RANSOD kit, from Randox Laboratories (UK). The assay principle employs xanthine and xanthine oxidase to generate superoxide radicals which react with 2-(4-iodophenyl)-3-(4nitrophenyl)-5-phenyltetrazolium chloride (INT) to form a red formazan dye. The superoxide activity was then measured by the degree of this reaction. One unit of enzyme activity is that which causes $50 \%$ inhibition of rate of reduction of INT under assay condition. Change in absorbance was recorded at $505 \mathrm{~nm} .{ }^{19}$

\section{GPx Activity Assay}

Blood GPx was measured using RANSEL kit from Randox Laboratories (UK). GPx catalyses the oxidation of glutathione (GSH) by cumene hydroperoxide. In the presence of GSH reductase and NADPH, the oxidized GSH is immediately converted to the reduced form with a concomitant oxidation of NADPH to $\mathrm{NADP}^{+}$. The decrease in absorbance at $340 \mathrm{~nm}$ was measured. ${ }^{20}$

\section{Vitamin C Assay}

The serum vitamin $\mathrm{C}$ content was measured using the dinitrophenylhydrazine (DNPH) method. ${ }^{21}$ In strong acidic medium, ascorbic acid is oxidized to diketogulonic acid which reacts with 2,4 DNPH to form diphenylhydrazine which dissolves in strong sulfuric acid solution to produce a red-colored complex which was measured at $500 \mathrm{~nm}$.

\section{MDA Assay}

The serum MDA was estimated according to the method of Kei S. ${ }^{22}$ Lipoproteins were precipitated from the serum by adding trichloroacetic acid. A total of $0.05 \mathrm{M}$ sulfuric acid and $0.67 \%$ thiobarbituric acid (TBA) in $2 \mathrm{M}$ sodium sulfate were added and the coupling of lipid peroxide with TBA was carried out by heating. The resulting chromogen was extracted in n-butanol whose absorbance was measured at $530 \mathrm{~nm}$.

\section{CRP Assay}

The serum CRP was measured by the quantitative latex turbidity method using the CRP-TURBI kit from Spinreact (Spain). The latex particles coated with specific anti-human CRP was agglutinated when mixed with samples containing CRP. The agglutination causes absorbance change (measured at $540 \mathrm{~nm}$ ), dependent upon the CRP content of the test sample that can be quantified by comparison from a calibrator of known CRP concentration. ${ }^{23}$

\section{Statistical Analysis}

The measured values for clinical parameters and biochemical markers at baseline and 3 months posttreatment were subjected to statistical analysis using statistical package for social sciences (SPSS version 11.5) for MS Windows. The values on clinical and biochemical parameters were expressed as mean \pm SD. Comparison of clinical and biochemical parameters across two study groups were done using independent sample ' $t$ ' test. In group II the effect of SRP (after 3 months post-treatment with the corresponding baseline parameters) on clinical and biochemical parameters was assessed using paired ' $t$ ' test.

The underlying normality assumption was tested for each clinical and biochemical parameter using percentilepercentile (PP) plot technique before applying respective ' $\mathrm{t}$ ' tests. A p-value $<0.05$ is considered to be statistically significant.

\section{RESULTS}

\section{Clinical Measurements}

The average clinical measurements are listed in Table 2. The average baseline clinical parameters are significantly higher $(\mathrm{p}<0.001)$ in chronic periodontitis patients (group II) compared to healthy controls (group I).

Following SRP therapy all clinical parameters reduced significantly ( $p<0.001$ ) compared to their corresponding baseline values in group II.

\section{Biochemical Studies}

The statistical comparison of biochemical parameters evaluated between the two study groups are shown in Table 3. The baseline parameters were found to be significantly different $(\mathrm{p}<0.001)$ in group II as compared group I. The average TAOC, GPx and vitamin C were significantly lower $(\mathrm{p}<0.001)$ and RBC-SOD, MDA and CRP were significantly higher $(\mathrm{p}<0.001)$.

Following SRP therapy all biochemical parameters were significantly improved $(\mathrm{p}<0.001)$ compared to the corresponding baseline values in group II. 
Table 2: Comparison of clinical parameters between groups I and II

\begin{tabular}{cccccc}
\hline Clinical parameters & Group I $(n=120)$ & & Group II $(n=82)$ & $\begin{array}{l}p \text {-value } \\
\text { group I vs }\end{array}$ & $\begin{array}{l}p \text {-value } \\
\text { group II } \\
\text { group II } \\
\text { baseline vs } \\
\text { post-treatment }\end{array}$ \\
\cline { 3 - 6 } & & Baseline & Post-treatment & 0.001 \\
GI & $0.7 \pm 0.1$ & $2.3 \pm 0.5$ & $1.5 \pm 0.5$ & 0.001 & 0.001 \\
PI & $0.4 \pm 0.2$ & $2.2 \pm 0.5$ & $1.3 \pm 0.5$ & 0.001 & 0.001 \\
PBI & $0.9 \pm 0.8$ & $2.6 \pm 0.4$ & $1.6 \pm 0.4$ & 0.001 & 0.001 \\
CAL (mm) & $1.8 \pm 0.3$ & $7.7 \pm 0.9$ & $6.2 \pm 1.0$ & 0.001 & \\
\hline
\end{tabular}

Values are mean $\pm \mathrm{SD}$; ${ }^{\mathrm{p}} \mathrm{p}$-values for mean $\pm \mathrm{SD}$ obtained by independent sample 't' test; ${ }^{\mathrm{b}} \mathrm{p}$-values for mean $\pm \mathrm{SD}$ obtained by paired 't' test; $p$-values $\leq 0.05$ is considered to be statistically significant

Table 3: Comparison of biochemical parameters between groups I and II

\begin{tabular}{|c|c|c|c|c|c|}
\hline \multirow[t]{2}{*}{ Biochemical parameters } & \multirow[t]{2}{*}{ Group I $(n=120)$} & \multicolumn{2}{|c|}{ Group II $(n=82)$} & \multirow{2}{*}{$\begin{array}{l}\text { p-value } \\
\text { group I vs } \\
\text { group II } \\
\text { (baseline) }\end{array}$} & \multirow{2}{*}{$\begin{array}{l}\text { p-value }{ }^{b} \\
\text { group II } \\
\text { baseline vs } \\
\text { post-treatment }\end{array}$} \\
\hline & & Baseline & Post-treatment & & \\
\hline TAOC $(\mu \mathrm{m} / \mathrm{l})$ & $913.3 \pm 67.3$ & $826.6 \pm 78.6$ & $910.0 \pm 69.4$ & 0.001 & 0.001 \\
\hline RBC-SOD (U/g Hb) & $292.5 \pm 37.8$ & $524.3 \pm 81.9$ & $439.6 \pm 75.9$ & 0.001 & 0.001 \\
\hline GPx (U/g Hb) & $13.7 \pm 1.4$ & $8.3 \pm 1.2$ & $9.6 \pm 1.1$ & 0.001 & 0.001 \\
\hline Vitamin $C(\mu \mathrm{m} / \mathrm{l})$ & $34.0 \pm 0.07$ & $22.7 \pm 0.07$ & $28.4 \pm 0.07$ & 0.001 & 0.001 \\
\hline $\mathrm{MDA}(\mathrm{nm} / \mathrm{ml})$ & $2.0 \pm 0.3$ & $4.1 \pm 0.4$ & $2.7 \pm 0.5$ & 0.001 & 0.001 \\
\hline CRP (mg/l) & $1.8 \pm 0.3$ & $3.4 \pm 0.4$ & $2.4 \pm 0.4$ & 0.001 & 0.001 \\
\hline
\end{tabular}

Values are mean $\pm \mathrm{SD}$; ${ }^{\mathrm{a}} \mathrm{p}$-values for mean $\pm \mathrm{SD}$ obtained by independent sample ' $\mathrm{t}$ ' test; ${ }^{\mathrm{b}} \mathrm{p}$-values for mean $\pm \mathrm{SD}$ obtained by paired ' $t$ ' test; $p$-values $\leq 0.05$ is considered to be statistically significant

\section{DISCUSSION}

The present study has focused on the relationship between periodontitis and systemic biochemical oxidative stress and inflammatory markers and effect of SRP therapy on these markers. It is suggested that patients with periodontal disease are more susceptible to an imbalance of antioxidantoxidative stress situation. ${ }^{1}$ Studies ${ }^{1,2,7}$ have associated altered clinical parameters in chronic periodontitis as compared to healthy controls. In the present study we have observed significantly higher GI, PI, PBI and CAL in group II at baseline compared to group I. The SRP has shown an improvement in all the clinical parameters after 3 months in group II compared to their respective baseline values. Similar effect of improvement in clinical parameters after SRP has also been reported by studies. ${ }^{11,24}$

Prospective studies ${ }^{2,5,25,26}$ have associated periodontitis with TAOC. TAOC reflects full spectrum of antioxidant activity against various reactive oxygen and nitrogen radicals. Generally, low TAOC indicates oxidative stress or increased susceptibility to oxidative damage. ${ }^{27}$ In accordance to our findings, studies ${ }^{25,26}$ have observed lower TAOC in subjects with chronic periodontitis as compared to the healthy controls. The nonsurgical periodontal treatment was effective at improving clinical parameters and reducing plasma reactive oxygen metabolites (ROMs) and ROS. ${ }^{28}$ As ROS is lowered after periodontal therapy an improvement in TAOC is expected, as we have observed in our study.
Antioxidant enzymes, SOD and GPx provides protection within the cell against ROS. ${ }^{29}$ Studies have reported higher ${ }^{1}$ and lower ${ }^{30}$ SOD activities in chronic periodontitis. The present study has observed significantly higher RBC-SOD activity in group II at baseline as compared to group I. The human periodontal ligament has shown to possess the enzyme SOD which offers biological protection against ROS. Bacterial lipopolysaccharides also stimulate superoxide release from gingival fibroblast, suggesting that the induction of SOD may represent an important defense mechanism of the fibroblasts during inflammation. Moreover, increased SOD in inflamed gingiva may indicate increased superoxide radical generation by PMNLs invading at the diseased sites. ${ }^{1}$ SRP therapy is aimed at reducing the disease causing microbes associated with oral plaque. This may help in decreasing the inflammatory manifestations. The decreased pathogen load and local inflammation may help in lowering super oxide radical generation and reducing the SOD enzyme activity as observed in our study.

The studies in the literature have reported insignificantly changed $^{31}$ and significantly lowered ${ }^{29}$ GPx activities in periodontitis patients compared to healthy controls. The present study has obtained lower GPx activity in chronic periodontitis group than in controls at baseline. The direct proportionality in GPx activity in gingival crevicular fluid (GCF) with the severity of periodontal disease has been reported. ${ }^{6}$ Al Rawi (2011) ${ }^{32}$ have reported that oxidative stress in various pathologies (including periodontitis) does 
not depend on a loss of reduced GSH or a lack of GSH synthesis alone but a misbalance in the oxidant/reductant cycle of GSH. The concentration of GSH in gingival cervicular fluid was compromised in chronic periodontitis and nonsurgical periodontal therapy resulted in nonsignificant increase in concentration of GSH and total GSH but the redox balance in terms of GSH:GSSG increased to that of periodontal healthy controls. ${ }^{24}$ The investigations in the above studies indicate that oxidative stress depleted GSH concentration and disturbed the GSH:GSSG balance. The depleted GSH concentration may be reflected in lowered GPx activity. The SRP therapy which may lead to increased GSH concentration due to improved GSH:GSSG balance may result in an increased activity of GPx, as we have observed in our study.

Vitamin C (ascorbic acid) is a low molecular weight, water soluble antioxidant. It has protective effect on maintaining tissue homeostasis by playing an important role in collagen synthesis and therefore helps in maintenance of structural integrity of the connective tissue. Vitamin C also has a beneficial role as radical scavenger. ${ }^{33}$ Through its antioxidant action, vitamin $\mathrm{C}$ neutralizes oxidative stress, and in doing so may be depleted in plasma. It is therefore quite possible that periodontitis causes lower plasma vitamin $\mathrm{C}$ through this mechanism. ${ }^{34}$ In our study serum vitamin $C$ level was lower in chronic periodontitis at baseline as compared to healthy controls, which was also documented by studies. ${ }^{34,35}$ Post-SRP therapy a significant improvement in plasma vitamin C levels was observed indicating lowered oxidative stress and improved antioxidant status.

Lipid peroxidation (LPO) has been implicated in the pathogenesis of several pathological disorders including periodontal disease. ROS can attack polyunsaturated fatty acids and induce formation of LPO products such as MDA. It is a stable end product of peroxidation of lipids by ROS. MDA is one of the most frequently used indicators of LPO and may be a potential biomarker indicating oxidative stress. ${ }^{36}$ Our finding indicates higher serum MDA level in chronic periodontitis at baseline as compared to the healthy controls which have been documented by many researchers ${ }^{1,31,37}$ in chronic periodontitis. Tsai et al (2005) ${ }^{31}$ have reported that LPO was significantly correlated with clinical parameters of periodontal disease, and periodontitis patients showed significantly lower LPO concentration after treatment than before therapy which is in accordance with our study. SRP therapy aids in lowering LPO which results in decreasing MDA concentration in serum of periodontal patients.

CRP is an acute phase protein, which is used as an inflammatory marker. It has been demonstrated by studies $^{12,38,39}$ that CRP levels are higher in periodontitis patients than in healthy subjects, which supports our findings. Renvert et al (2009) ${ }^{39}$ have observed that periodontal treatment along with anti-inflammatory treatment resulted in a significant decrease in hs-CRP and proinflammatory cytokines in subjects with periodontitis. Another study by Vidal et al (2009) ${ }^{40}$ has reported that nonsurgical periodontal therapy was effective in improving periodontal clinical data and in decreasing the plasma levels of IL6, CRP and fibrinogen in hypertensive patients with periodontitis. The result of our study is in accordance with the above studies. The result of the present study indicates improved CRP levels after SRP therapy. This improved CRP (marker for systemic inflammation) level could be attributed to the beneficial effects of SRP therapy, which lowers the inflammatory burden on the periodontium.

The balance between ROS and antioxidant defense mechanism is likely to be important in periodontal pathogenesis. As observed in our study, patients with chronic periodontitis showed higher inflammatory and oxidative stress markers and lower antioxidant defense at baseline. SRP procedures belong to the initial phase of an orderly sequence of periodontal treatment. The primary objective of SRP therapy is to completely remove the gingival inflammation provoking elements (i.e. plaque, calculus, endotoxins) from the tooth surface. In doing so, the therapy reduces inflammation and helps in improving the host defense by restoring the disturbed balance of oxidant: antioxidant. The restoration of oxidant: antioxidant balance may help to delay/or control the onset of various systemic inflammatory diseases; at least those initiated by periodontitis. We recommend further longitudinal studies to establish the findings of the present study and also to assess the efficacy of periodontal therapy in various systemic diseases associated with periodontitis.

\section{CONCLUSION}

Patients with chronic periodontitis show higher clinical periodontal damage, systemic oxidative stress and inflammation compared to healthy controls. Clearly, the study demonstrates the efficacy of SRP in effectively eliminating the etiologic agent, thereby helping in decreasing the inflammatory impact on the periodontal tissues and subsequently improving clinical parameters and systemic biochemical oxidative stress and inflammatory markers. The prevention and treatment of periodontitis using SRP therapy may be a useful support in the control of the various chronic diseases associated with inflammation.

\section{ACKNOWLEDGMENTS}

Authors are thankful to Professor Salma Aziz, Mr Javed Akhtar for funding the study and Dr KJ Namazi, 
Dr S Sumanth, Dr R Kale, Mr Mehmood S and Mr Pankaj for the technical support in conducting the study.

\section{REFERENCES}

1. Wei D, Zhang XL, Wang YZ, Yang CX, Chen G. Lipid peroxidation level, total oxidant status and super oxide dismutase in serum, saliva and gingival cervicular fluid in chronic periodontitis patients before and after periodontal therapy. Aust Dent J 2010;55(1):70-78.

2. Chapple ILC, Brock GR, Milward MR. Compromised GCF TAOC in periodontitis: Cause or effect. J Clin Periodontol 2007;34(2):103-10.

3. Kim J, Amar S. Periodontal disease and systemic conditions: A bidirectional relationship. Odontology 2006;94:10-12.

4. Giustanini D, Dalle-Donne I, Tsikas D, Rossi R. Oxidative stress and human diseases: Origin, link, measurement, mechanism, and biomarkers. Critical Reviews in Clinical Lab Sciences 2009;46(5-6):241-81.

5. D’ Aiuto F, Nibali L, Parker M, Patel K, Suran T, Donos N. Oxidative stress, systemic inflammation and sever periodontitis. J Dent Res 2010;89(11):1241-46.

6. Patel SP, Pradeep AR, Chowdhry S. Cervicular fluid levels of plasma glutathione peroxidase (eGPx) in periodontal health and disease. Arch Oral Biol 2009;54(6):543-48.

7. Akalin FA, Toklu E, Renda N. Analysis of SOD activity levels in gingival and GCF in patients with chronic periodontitis and periodontally healthy controls. J Clin Periodontol 2005;32(3):238-43.

8. Chapple IL, Mathews JB. The role of reactive oxygen and antioxidant species in periodontal tissue destruction. Periodontol 2000;2007;43:160-232.

9. Djordjevic VB. Free radicals in cell biology. Int Rev Cytol 2004;237:57-89.

10. Valko M, Leibfritz D, Moncol J, Cronin MT, Mazur M, Telser J. Free radicals and antioxidants in normal physiological functions and human disease. Int J Biochem Cell Biol 2007;39:44-84.

11. Marcaccini AM, Meschiari CA, Zuardi LR, et al. Gingival crevicular fluid levels of MMP-8, MMP-9, TIMP-2, and MPO decrease after periodontal therapy. J Clin Periodontol 2010;37:180-90.

12. Gani DK, Lakshmi D, Krishnan R, Emmadi P. Evaluation of CRP and interleukins- 6 in the peripheral blood of patients with chronic periodontitis. J Indian Soc Periodontol 2009;13(2): 69-74.

13. Armitage GC. Development of a classification system for periodontal disease and conditions. Ann Periodontol 1999;4: 1-6.

14. Armitage GC. The complete periodontal examination. Periodontol 2000;2004;34:22-33.

15. Loe H, Silness J. Periodontal disease in pregnancy I, Prevalence and severity. Acta Odontol Scand 1963;21:533-51.

16. Silness J, Loe H. Periodontal disease in pregnancy II. Correlation between oral hygiene and periodontal condition. Acta Odontol Scand 1964;22:121-35.

17. Muhlemann HR. Psychological and chemical mediators of gingival health. J Prev Dent 1977;4:6-17.

18. Benzie Iris FF, Strain JJ. The ferric reducing ability of plasma (FRAP) as a measure of antioxidant power-the FRAP assay. Analytical Biochemistry 1996;239(1):70-76.
19. Woolliams JA, Wiener G, Anderson PH, McMurray CH. Variation in the activities of glutathione peroxidase and superoxide dismutase and in the concentration of copper in the blood in various breed crosses of sheep. Research in Veterinary Science 1983;34:253-56.

20. Paglia DE, Valentine WN. Studies on the quantitative and qualitative characterization of erythrocyte glutathione peroxidase. J Lab Clin Med 1967;70:158-69.

21. Harold V. Carbohydrates. In: Practical clinical biochemistry. London: CBS Publishers: London 2005:173-76.

22. Kei S. Serum lipid peroxide in cerebrovascular disorders determined by a new colorimetric method. Clinica Chemica Acta 1978;90:37-43.

23. Hanson LO, Lindquist L. C-reactive protein: Its role in the diagnosis and follow-up of infectious diseases. Curr Opinion Infect Dis 1997;10:196-201.

24. Grant MM, Brock GR, Mathews JB, Chapple ILC. Cervicular fluid glutathione levels in periodontitis and the effect of nonsurgical therapy. J Clin Periodontol 2010;37:17-23.

25. Konopka T, Krol K, Kopec W, Gerber H. Total antioxidant status and 8-hydroxyl-2-deoxyguanosine levels in gingival and peripheral blood of periodontitis patients. Arch Immunol Ther Exp 2007;55(6):417-22.

26. Canakci V, Yildrim A, Canakci CF, Eltas A, Cicek Y, Canakci $\mathrm{H}$. Total antioxidant capacity and antioxidant enzymes in serum, saliva and gingival cervicular fluid of pre-eclamptic women with and without periodontal disease. J Periodontol 2007;78(8): 1602-11.

27. Young IS. Measurement of total antioxidant capacity. J Clin Pathol 2001;54:339.

28. Tamaki N, Tomofuji T, Ekuni D, Yamanaka R, Yamamoto T, Morita M. Short-term effect of nonsurgical periodontal treatment on plasma level of reactive oxygen metabolites in patients with chronic periodontitis. J Periodontol 2009;80(6):901-06.

29. Canakci CF, Cicek Y, Yildirim A, Sezer U, Canakci V. Increased levels 8-hydroxyl-2-deoxyguanosine and MDA and its relationship with antioxidant enzymes in saliva of periodontitis patients. Eur J Dent 2009;3(2):100-06.

30. Baltacioqlu E, Aklain FA, Alver A, Balafan F, Unsal M, Karabulut E. Total antioxidant capacity and SOD activity levels in serum and GCF fluid in postmenopausal women with chronic periodontitis. J Clin Periodontol 2006;33(6):385-92.

31. Tsai CC, Chen HS, Chen SL, et al. Lipid peroxidation: A possible role in the induction and progression of chronic periodontitis. J Periodontol Res 2005;40(5):378-84.

32. Al Rawi NH. Oxidative stress, antioxidant status and lipid profile in the saliva of type 2 diabetics. Diabetes and Vascular Disease Research 2011;8(1):22-28.

33. Chapple ILC, Milward MR, Dietrich T. The prevalence of inflammatory periodontitis is negatively associated with serum antioxidant concentrations. J Nutr 2007;137:657-64.

34. Anwar TM. Plasma vitamin C is inversely associated with periodontitis. J Evid Base Dent Pract 2008;8:103-04.

35. Thomas B, Kumari S, Ramitha K, Ashwini Kumari MB. Comparative evaluation of micronutrients in the serum of diabetes mellitus patients and healthy individuals with periodontitis. J Indian Soc Periodontal 2010;14(1):46-49.

36. Nielsen F, Mikkelson BB, Nicholson JB, Anderson HR, Grandjean P. Plasma MDA as biomarker and oxidative stress: Reference interval and effects of life style pattern. Clin Chem 1997;43:1209-14. 
37. Khalili J, Bilokytska HF, Salivar Khalili J, Bilokytska HF. Salivary MDA levels in clinically healthy and periodontal diseased individuals. Oral Dis 2008;14(8):754-60.

38. Thakare KS, Deo V, Bhongade ML. Evaluation of CRP serum levels in periodontitis patients with or without atherosclerosis. Indian J Dent Res 2010;21(3)326-29.

39. Renvert S, Lindahl C, Jansaker AMR, Lessem J. Short term effects of an anti-inflammatory treatment on clinical parameters and serum levels of C-reactive protein and proinflammatory cytokines in subjects with periodontitis. J Periodontol 2009;80(6):892-900.

40. Vidal F, Figueredo CMS, Cordovil I, Fischer RG. Periodontal therapy reduces plasma levels of interlukin-6, C-reactive protein and fibrinogen in patients with severe periodontitis and refractory arterial hypertension. J Periodontol 2009;80(5):786-91.

\section{ABOUT THE AUTHORS}

\section{Abdul S Aziz (Corresponding Author)}

$\mathrm{PhD}$ Student, Department of Biochemistry, Grant Medical College and Sir JJ Group of Hospitals, Mumbai, Maharashtra, India, Phone:+919823375529 Fax: +91-020-26430962, e-mail: samadaziz79@ gmail.com

\section{Madhav G Kalekar}

Associate Professor, Department of Biochemistry, Grant Medical College and Sir JJ Group of Hospitals, Mumbai, Maharashtra, India

\section{Tabita Benjamin}

Head, Department of Dentistry, Grant Medical College and Sir JJ Group of Hospitals, Mumbai, Maharashtra, India

\section{Adinath N Suryakar}

Registrar, Maharashtra University of Health Sciences, Nashik Maharashtra, India

\section{Milsee M Prakashan}

Assistant Professor in Biochemistry, Department of Biotechnology Sinhgad College of Science, Ambegaon (BK), Pune, Maharashtra, India

\section{Mohammed Nadeem A Bijle}

Postgraduate Student, Department of Pedodontics, MA Rangoonwala College of Dental Sciences and Research Center, Pune, Maharashtra, India 